\title{
Uso de las TIC en tres facultades de comunicación ecuatorianas
}

\author{
César Ulloa \\ cesar.ulloa@udla.edu.ec

\begin{abstract}
Carrera de Comunicación Corporativa, Facultad de Comunicación y
\end{abstract} \\ Artes Audiovisuales, Universidad de Las Américas (Ecuador). \\ Fecha de finalización del trabajo: 3 de enero de 2020 \\ Recibido: 11 de enero de 2020. \\ Aceptado: 11 de mayo de 2020. \\ DOI: https://doi.org/10.26422/aucom.2020.0901.ull
}

\section{Resumen}

Los estudios acerca del uso e impacto de internet y de las tecnologías de la información y la comunicación (TIC) en la formación de los estudiantes de comunicación en el Ecuador son embrionarios, más aún desde un ejercicio de comparación que pudiera dar cuenta de las similitudes y las diferencias entre los planes académicos de las universidades cuando problematizan en profundidad las características de la sociedad de la información, el conocimiento, el mundo digital y las nuevas generaciones. Este artículo explora la realidad de las tres universidades que cuentan con el mayor número de estudiantes de comunicación en la ciudad de Quito y responde qué papel juegan la red y las TIC en la formación teórica, práctica y social. Esta propuesta sobrepasa la asociación instrumental entre el aprendizaje de la comunicación con el uso de las tecnologías informáticas e, incluso, el de las telecomunicaciones para producir y circular productos digitales. En ese sentido, entre los retos de la universidad en la formación de los estudiantes de comunicación se encuentra la dotación de sentido a las tecnologías y su uso en una sociedad de la información y el conocimiento que demanda respuestas no solo rápidas, sino más bien sostenidas de contenido y rigor.

Palabras clave: facultades de comunicación, oferta académica, enseñanza de TIC, Ecuador.

\section{Use of ICTs at three Schools of Communication in Ecuador}

\section{Abstract}

The study of the use and impact of the internet and of information and communication technologies (ICTs) in the instruction of communication students in Ecuador is at an embryonic stage. This is even more evident when we seek to compare and contrast the academic programs and curricula put forth by different universities and how they problematize aspects of the information and knowledge society, the digital world, and emerging generations. This article looks at the three universities in the city of Quito with the greatest number of communication students, and reveals the role played by the web and by ICTs in theoretical, practical, and social education. Our approach moves beyond the merely instrumental link between the study of communication and the use of information technologies and telecommunications to produce and circulate 
digital products. Among the challenges faced by universities, in educating their communication students, is the need to bestow meaning upon technologies and their use in an information and knowledge society - a need that demands a response that is both immediate and based on rigorous evidence.

Keywords: schools of communication, academic programs, teaching ICTs, Ecuador.

\section{Equador: uso das TIC nas faculdades de comunicação}

\section{Resumo}

Os estudos sobre o uso e o impacto da internet e das tecnologias da informação e a comunicação (TIC) na formação dos estudantes de comunicação no Equador são embrionários, mais ainda desde um exercício de comparação que poderia dar conta das semelhanças e as diferenças entre os planos acadêmicos das universidades quando problematizam a fundo as características da sociedade da informação, o conhecimento, o mundo digital e as novas gerações. Este artigo explora a realidade das três universidades com o maior número de estudantes de comunicação na cidade de Quito e responde qual é o rol da rede e das TIC na formação teórica, prática e social. Essa proposta ultrapassa a associação instrumental entre o aprendizado da comunicação com o uso de tecnologias de computador e mesmo o de telecomunicações para produzir e circular produtos digitais. Nesse sentido, entre os desafios da universidade na formação dos estudantes de comunicação está a provisão de significado para as tecnologias e seu uso em uma sociedade de informação e conhecimento que exige respostas não apenas rápidas, mas sustentadas de conteúdo e rigor.

Palavras chave: faculdades de comunicação, oferta acadêmica, ensino de TIC, Equador.

\section{Introducción}

Es inevitable el uso de internet, de las tecnologías de la información y la comunicación (TIC) y de las redes sociales en la sociedad del conocimiento, debido a que la mayoría de las actividades cotidianas están caracterizadas por el intercambio de intangibles en sus distintos formatos digitales. Dentro de la red, bajo una lógica online, se transporta una cantidad incuantificable de datos sin límites de espacio ni tiempo, desde los cuales se hacen realidad transacciones financieras y bancarias, se compran y venden productos, se contratan servicios, se gestan procesos de deliberación pública sobre alguna política local, nacional e internacional (Bianchi, 2017), se propician diversos tipos de activismos y se fomenta la participación masiva en cursos de formación técnica bajo una mirada autodidacta e interactiva y de acceso gratuito.

Internet y las TIC han transformado la vida de las personas y de las situaciones en los siguientes aspectos, aunque no sean los únicos ni exclusivos: a) una nueva forma de habitar el mundo e incluso de resignificar la idea del poder "tradicional" (Castells, 2009; Durán Barba y Nieto, 2017; Naím, 2013); b) la innovación pedagógica en todos los niveles debido a la intensificación en el uso de recursos audiovisuales (Apolo, Bayés y Hermann, 2016; Cassany y Ayala, 2008; García, González y Ramos, 2010; González y De Pablo Pons, 2015; Linne, 2014); c) la emergencia de nuevas generaciones, como los 
nativos digitales en el segmento de los millennials y centennials, quienes tienen sus propias demandas y construcción de sentidos (Gutiérrez-Rubí, 2016; Ulloa et al., 2018a); y d) el mundo del trabajo, las ventajas competitivas y las competencias tecnológicas (Yoguel, 2014), entre otros.

La sociedad del conocimiento replanteó los procesos de enseñanza y aprendizaje en todos los niveles de formación educativa (Buckingham, 2007; Ferrés y Piscitelli, 2012; Hooper y Rieber, 1995; Salinas, 2004). Las causas de esta transformación a escala global se explican gracias a las características de las TIC, el acceso a los dispositivos informáticos, la relación entre las distintas generaciones cuando se trata de analizar el mismo objeto de estudio desde diferentes perspectivas, las demandas del mercado laboral y las amenazas de la humanidad, que van desde lo climático pasando por el crimen transnacional organizado, la corrupción y las nuevas maneras de gestionar la política. En este contexto, las universidades están expuestas a revisar de manera permanente los planes de estudio, las metodologías, las competencias, los recursos, la formación de los docentes y las demandas de los estudiantes para responder a las exigencias de un mundo cambiante y competitivo.

Con la finalidad de analizar el contexto mencionado con un objeto de estudio, a continuación se plantea responder la siguiente pregunta en correspondencia con los retos que la sociedad contemporánea exige a la educación superior en la enseñanza de la comunicación: ¿de qué manera, en Ecuador, las carreras de comunicación de la Universidad de Las Américas (UDLA), la Universidad Central del Ecuador (UCE) y la Universidad Tecnológica Equinoccial (UTE) conciben la enseñanza de las TIC en la sociedad del conocimiento? A tal efecto, se describirá y explicará cómo las carreras de comunicación han planteado una estrategia curricular (conceptual, metodológica, evaluativa y práctica) de cara a la formación de sus estudiantes en dos aspectos: la comprensión de los elementos que configuran la sociedad contemporánea y el uso de todos los artilugios tecnológicos para la inserción de sus estudiantes en el mundo digital. En la sección de metodología, se precisa el objetivo de la investigación, la selección de los casos y la obtención y el procesamiento de los datos.

La organización de este artículo se basa en la obtención de los resultados de una dimensión del proyecto “Tecnología, jóvenes y educación”, que llevó a cabo la carrera de Comunicación Corporativa de la UDLA desde 2015 hasta 2017 y que ha extendido el debate en la comunidad universitaria hasta 2019. La dimensión gira alrededor del análisis comparativo de las mallas curriculares de las tres carreras de las tres universidades con énfasis en el uso de las TIC, de los criterios de las autoridades académicas mediante el procesamiento de entrevistas en profundidad y de los datos obtenidos de una encuesta que se llevó a cabo a los estudiantes de las universidades. La metodología se inscribe en una lógica de triangulación de datos de carácter cualitativo y cuantitati- 
vo, bajo el uso de fuentes primarias y secundarias y de la documentación oficial de los casos de estudio acerca de la planificación curricular.

Los alcances de este artículo responden a una propuesta de investigación exploratoria y comparada que abre las puertas a un debate más amplio sobre cómo las carreras de comunicación en Ecuador conciben el perfil de egreso de los estudiantes con respecto a la sociedad del conocimiento. Los límites advierten la imposibilidad de generalizar los resultados a una realidad geográfica más amplia, sin embargo, dan cuenta de las similitudes y de las diferencias que podrían reiterarse en otros centros de educación superior. Al final, se exponen los hallazgos de cara a un hecho concreto: lo inevitable que significa para un estudiante de comunicación comprender y ejercer su profesión a partir de los retos, exigencias y demandas de una realidad híper comunicada, en la que el conocimiento es poder.

\section{Superar la mirada fragmentaria}

Este artículo escapa de la prototípica discusión que divide a la sociedad en alfabetos y analfabetos digitales, tecnófilos y tecnófobos, nativos e inmigrantes digitales (Prensky, 2001); más bien, da cuenta de que el contexto de análisis es más complejo cuando se abordan los hábitos de consumo de internet y de las TIC. ${ }^{1}$ No se trata de hacer un ejercicio en el que se coloca a las personas en los extremos; tampoco de reducir la realidad educativa a una esfera con fines instrumentales o a otra con afanes humanísticos (Bisbal, 2001; Ficarra, 2001). Aquí, se sobrepasa la idea de demonizar, celebrar o mitigar a quienes no hacen uso de las tecnologías, a quienes hacen uso parcial o casi total de estas. En cambio, se subraya que las personas en la sociedad del conocimiento caminan a diferentes ritmos y pueden observar los mismos fenómenos con sentidos diversos. La propuesta de salir de la dicotomía se basa en que un ejercicio de comparación entre tres universidades no tiene como objetivo concluir en términos de superioridad/inferioridad de unas hacia otras en cuanto al uso, acceso y hábitos a la tecnología, más aún en casos distintos. El objetivo es comprender cómo conciben las universidades, que se utilizan como casos de estudio, la enseñanza de las TIC en la sociedad del conocimiento.

Con la finalidad de precisar y delimitar los conceptos que se utilizan aquí, adelante se exponen los elementos constitutivos de la sociedad del conocimiento, los jóvenes que forman parte del estudio y que se inscriben en lo que ahora se conoce como "Generación Y" o millennials (Piscitelli, 2009; Varela, 2012), el mundo digital (Negroponte, 1995), la sociedad red (Castells, 2009), las competencias mediáticas

\footnotetext{
Para Prensky (2001), las características de los nativos digitales son la adicción a la tecnología, la idea de que la vida va de la mano del funcionamiento de las TIC: velocidad, respuesta inmediata, interacción. El riesgo de sufrir nomofobia (no mobile phobia) o los síntomas de trastorno que puede provocar el uso adictivo de los celulares.
} 
(López y Aguaded, 2015) y los modelos de apropiación de las TIC (Sandia, Luzardo y Aguilar-Jiménez, 2019). Estos conceptos no están aislados, pues se auxilian y se complementan bajo la perspectiva de caracterizar al mundo contemporáneo, que para muchos evidencian liviandad y liquidez en la forma de pensar, hacer y vivir (Bauman, 2006), sino, y sobre todo, porque el cambio es una regularidad y no algo excepcional (Ulloa, 2014). También se habla de que la escuela está atravesada por la incertidumbre (Rodríguez de las Heras, 2008), en el sentido de que no hay un criterio último acerca de la influencia que pueden tener las TIC en los procesos de formación, ya que una experiencia exitosa no es necesariamente transferible y, por otro lado, hay condiciones que aceleran o retardan la incorporación de las TIC en la educación, como la brecha digital, la inversión en infraestructura, el personal especializado y la idea de dar paso a una cultura digital.

Este primer momento permite contextualizar en qué sociedad se desenvuelven los jóvenes y después abre el paso a un segundo para contrastar estos criterios con los planes de estudio de las carreras de comunicación de las universidades, para así conocer si estas propuestas logran insertar a los estudiantes en la sociedad del conocimiento desde lo conceptual, lo práctico y lo social. Cabe precisar que la exploración analítica de la oferta académica de las universidades como dimensión de estudio no es nueva, ya que se encuentra en investigaciones orientadas a la pedagogía, la relación entre la oferta académica y el mercado laboral y el rediseño de los planes de estudio. ${ }^{2}$

La sociedad del conocimiento a la cual se hace alusión se caracteriza por cambiar el concepto de "riqueza" (Tofler y Tofler, 2006), ya que el pensamiento elaborado es un intangible que puede tener un valor incuantificable. Se pone en evidencia la vertiginosa actualización y producción de conocimientos que circulan por la red con accesos gratuitos y también pagos; la velocidad con la que circula la información se manifiesta con prácticas de interacción entre los sujetos sin importar su lugar de origen y localización, pero este hecho abre la posibilidad de generar asimetrías en la competencia entre los sujetos, empresas e instituciones por el acceso desigual a la información. En la sociedad del conocimiento, los recursos didácticos -así como la pedagogía- se reinventan en la medida en que gana terreno lo audiovisual, la consulta en portales de internet, el consumo ágil de la información y la mediación virtual entre docentes y estudiantes (Whitby, 2013); sin embargo, pensar que la sociedad digital por sí sola cambia

\footnotetext{
2 A modo de ejemplo, para el caso de la región Andina, en lo que se refiere a la enseñanza y uso de las TIC en las carreras de comunicación, hay evidencias de estudios en Colombia (Trillos, 2010) y Ecuador (Punín, 2012; Punín, Rivera y Marín, 2014), entre otros. En los primeros, se trata de comprobar de qué manera el cambio que sufrió la malla curricular de la Universidad Autónoma del Caribe (Barranquilla) redujo la capacidad lectoescritora de los estudiantes, debido a un enfoque instrumental que privilegia la producción de piezas comunicacionales antes que el pensamiento crítico. En los estudios de Ecuador, se enfatiza en la ausencia de una malla curricular que forme a los estudiantes para la comunicación digital, así como también se resalta que los periodistas se forman en la "calle" antes que en las aulas, por lo cual, habría un divorcio entre la oferta académica y las demandas del mercado laboral.
} 
y mejora la realidad en términos de calidad de vida en lo que respecta al aprendizaje es una lectura muy simplificada y descontextualizada (González y De Pablo Pons, 2015).

La sociedad red, según Castells (1997), no es otra cosa que la interacción e interdependencia inevitables entre las diferentes economías de un mundo globalizado que modela las prácticas de los individuos, a partir de una estructura que el autor considera como dominante. La interacción se produce desde dinámicas de deslocalización y desconcentración, es decir, no hay un lugar específico desde donde se producen las cosas y los relatos y, peor aún, no hay un centro en donde se hagan todas las actividades de la producción. La interacción se produce por los avances de la tecnología en el campo de las telecomunicaciones, situación que acerca, mixtura y pone en diálogo a las culturas.

En lo que concierne al mundo digital, Negroponte (1995) advirtió que las bondades de la tecnología en términos de capacidad de almacenamiento, software, cobertura y movilidad no traen consigo una homogeneización en el consuno de información, ya que la "posinformación" pone en evidencia un intento de segmentar cada vez más a la población en distintas audiencias desde propuestas individualizadas.

Las TIC atraviesan todas las actividades en las esferas pública y privada. Son omnipresentes porque no se puede prescindir de ellas al planear y hacer la vida cotidiana. De ahí la emergencia de conceptos como "tecnología como entorno relacional" (Acedo, Lazo y Aparici, 2013), porque las TIC determinan las maneras en cómo los sujetos construyen la sociedad en el intercambio de ideas, emociones, saberes y conocimientos. Son, por lo tanto, medios y herramientas para establecer acercamientos online entre los sujetos, aun cuando haya entre ellos anonimato por la multiplicidad de facetas que puede asumir un internauta en las redes.

En este escenario, resulta ineludible mencionar las principales características de la Web 2.0, siguiendo los aportes de Acedo, Lazo y Aparici (2013), quienes, a su vez, hacen uso de los criterios de otros autores: a) la "inteligencia colectiva", es decir, se diluye la idea de que la propiedad del conocimiento es monopólica, sino más bien que todo es el resultado de un acumulado histórico con la participación activa de múltiples personas que están pensando simultáneamente; b) "multitudes inteligentes" (Rheingold, 2004), que significa la coexistencia de varias personas que actúan sin una organización explícita y logran propósitos coyunturales, situación que puede ser favorable o no en función de qué se trata de conseguir; c) la "intercreatividad" (Berners-Lee y Fischetti, 2000), que es la creación conjunta a través de la interacción que se produce en la red entre sujetos que no tienen que conocerse, pero sí actuar sobre la base de intereses comunes; y d) nuevas estructuras de participación, en las que se trata de gestionar modelos con mayor democracia.

La importancia que ha cobrado en el mundo la producción, circulación y consumo de información digital de manera instantánea y que viene en diversos formatos -de- 
bido a la transición provocada por internet entre los medios tradicionales a los digitales- pone en debate si la población está capacitada para adaptar y apropiarse de una nueva pedagogía de consumo comunicacional, es decir, no solo de acceder a las TIC, sino también de procesar la información o diseñar filtros para distinguir la información verdadera de la falsa, la de baja y alta calidad, la firmada y la anónima, las noticias elaboradas y las fake news (noticias falsas). Esta realidad reaviva el debate acerca de implementar en las aulas de los distintos niveles de formación educativa asignaturas que desarrollen las competencias mediáticas (Ferrés y Piscitelli, 2012; López y Aguaded, 2015), que implica educar para producir y también para consumir información con contenido plural, real, democrático, inscrito en los derechos humanos, inclusivo y ético, cuando también "un proceso por el que las personas llegan a conseguir una educación tecnológica y mediática” (Marín, Rivera y Celly, 2014, p. 121).

Para ampliar el concepto de "competencia mediática", a continuación se describen los aportes de López y Aguaded (2015) en los elementos que considero importantes para la investigación:

Lenguaje: conocimiento de los códigos, capacidad para utilizarlos y para analizar mensa-
jes escritos y audiovisuales desde la perspectiva del sentido y el significado, de las estruc-
turas narrativas y de las categorías y géneros. Tecnología: conocimiento y capacidad de
utilización de las herramientas que hacen posible la comunicación escrita y audiovisual
para entender cómo se elaboran los mensajes. Comprensión del papel que desempeñan
las TIC en la sociedad. Procesos de producción y difusión: conocimiento de las funciones
y tareas de los agentes de producción, las fases de los procesos de producción y difusión
y los códigos de regulación. Capacidad para elaborar, seleccionar, compartir y diseminar
mensajes mediáticos (p. 189).

Esto significa que el docente y el estudiante de comunicación requieren una preparación para responder a las exigencias de la sociedad del conocimiento. Es una situación de doble vía que compromete responsabilidades, destrezas y conocimientos de parte de los dos actores. Como ya se ha manifestado, la enseñanza de las TIC que sobrepasa la mirada instrumental y se concentra en la calidad de los contenidos requiere el análisis de los siguientes aspectos: a) formación y preparación de los docentes; b) planes de las carreras de comunicación; c) perfiles de egreso; y d) aptitudes y actitudes de los estudiantes.

Respecto a la formación y preparación de los docentes en el manejo de las TIC y su vinculación con el desarrollo de las competencias mediáticas, en los últimos años se han propuesto varios modelos para identificar en qué momento de apropiación están los docentes y las implicaciones en el escenario de enseñanza y aprendizaje (Hooper y Rieber, 1995; Sandia, Luzardo y Aguilar-Jiménez, 2019; Unesco, 2011). Estos modelos coinciden en varios aspectos, en el sentido de que hay fases de aprendizaje: una inicial, una intermedia y una de experticia. 


\begin{abstract}
Para Adell (2008), el proceso de apropiación contempla 5 niveles. Se inicia con el nivel acceso, en el que los docentes aprenden el uso básico de la tecnología. Luego viene la etapa de adopción, donde simplemente ellos usan la tecnología para hacer lo mismo que hacían antes. Se pasa a la siguiente fase o nivel de adaptación, en el que la nueva tecnología es integrada en prácticas docentes tradicionales, pero aumentando la productividad. En la fase de apropiación, se comienzan a experimentar nuevos usos de la tecnología, haciendo cosas que antes no hacían. Hasta llegar al nivel de innovación, cuando se usa la tecnología de una manera tal que antes nadie lo había realizado, es un acto creativo (Sandia, Luzardo y Aguilar-Jiménez, 2019, p. 273).
\end{abstract}

Como se puede notar, la sociedad del conocimiento demanda la comprensión de un contexto multifactorial, en el que si bien el uso de las tecnologías informáticas y las telecomunicaciones explican varias transformaciones en los procesos de relacionamiento social, hay que ir más allá, sobre todo en lo que se hace desde la educación para conocer si aquello que se propone no se circunscribe únicamente al uso instrumental de computadoras, teléfonos móviles, tabletas y los demás artilugios, sino más bien a la dotación de contenido a la tecnología.

A diferencia de otras épocas, el nuevo siglo introdujo una variable demográfica que coindice con los avances de las TIC y de las telecomunicaciones y que se expresa en su mayor auge con la sociedad red. En todo el mundo, se produjo un cambio generacional con un segmento de la población de jóvenes conocidos como millennials o "Generación Y". Esta definición fue creada por los demógrafos Neil Howe y William Strauss, quienes identifican a los jóvenes que nacieron a inicios de la década de los 80 y los cinco primeros años de los 90 . Este segmento de la población presenta las siguientes características: son nativos digitales, herederos de la globalización, viven en democracia porque no conocieron la dictadura, sus relaciones se gestan en las redes sociales y persiguen causas como la defensa del medio ambiente, los derechos de los animales y la defensa de las diversas preferencias sexuales (Ulloa, 2018a; 2018b).

A manera de referencia, el segmento de los millennials representa el $30 \%$ de personas en América Latina. Para el caso de Ecuador, "casi 5 millones tenían entre 15 y 34 años en 2010, lo que significa que, aproximadamente, uno de cada tres ecuatorianos era, por ese entonces, un millennial' (Gutiérrez-Rubí, 2016, p. 2). Cabe aclarar que el último censo que se realizó en Ecuador fue en 2010 y el próximo se realizará en 2020. La última proyección que hizo el Instituto Nacional de Estadística y Censos (INEC, 2017) determinó que hay 3,9 millones de millennials, de los cuales el 50,4\% son mujeres y el $49,6 \%$ son hombres. Entre los datos sobre el acceso y uso de tecnologías, resulta que sesenta y cinco de cada cien personas tienen un smarthphone, el $68,7 \%$ usa internet y el $63,8 \%$ tiene al menos una red social.

En la sociedad del conocimiento, la mayoría de la población, en términos etarios, está dentro de la Generación Y. Este segmento requiere para su relacionamiento social un conjunto de competencias mediáticas que le permitan comprender la producción 
y circulación de relatos en el universo de internet y, específicamente, en las redes sociales, donde ocupa un gran porcentaje de su tiempo. La disciplina que más acerca a reflexionar este fenómeno es la comunicación, de ahí la importancia de conocer cómo las universidades están procesando los cambios del mundo desde aspectos concretos, como el diseño de los planes de estudio y el tipo de profesional que van a graduar, los recursos tecnológicos con que se cuenta, la formación de los docentes y el nivel en el que se encuentran y el criterio de los estudiantes. El uso de los recursos de internet, así como de las TIC, no dependerá solo de lo que enseñan los profesores, sino también de la apropiación que cada cual haga de estos. En la red, cada persona se pone su propio límite de navegación en profundidad, pero también en el tiempo de acceso.

Castells $(1997 ; 2009)$ ya advirtió sobre un nuevo tipo de sociedad, en la que la estructura dominante surgió a partir de un modelo que propicia la interacción entre la economía, el Estado y la sociedad; sin embargo, el mundo globalizado no es sinónimo de homogeneización (Negroponte, 1995). Estas tensiones no resueltas de un mundo que globaliza, pero que no homogeneiza, ponen en discusión lo que Naím (2013) vaticina en cuanto al fin del poder o la revolución de la riqueza por el poder que toma el conocimiento. Y todos estos factores están ligados con la sociedad del conocimiento, la comunicación y la información, es decir, todas sus demandas y exigencias. Aquí, el papel que juega la universidad es inevitable para desentrañar los cambios permanentes que se producen, sobre todo desde las carreras de comunicación, que tienen, entre sus objetos de estudio, la configuración de los relatos y la creación de imaginarios.

Con la finalidad de conocer cómo las carreras de comunicación hacen frente a todos estos escenarios cambiantes, se diseñó una estrategia metodológica que se expone a continuación, sin perder de vista que el perfil del actual comunicador lo convierte en un actor clave en la medida que traduce lo complejo en sencillo, realiza piezas comunicacionales multiformato, posiciona ideas, instituciones o personas, maneja crisis en sus diversas modalidades y entrena en vocería para la actuación eficiente en los medios masivos, las redes sociales y las TIC, pero, además, introduce una nueva pedagogía en la que incluye el entretenimiento. Y todo ello se enmarca en el conocimiento y puesta en las aulas de las competencias comunicativas.

\section{Análisis de las ofertas académicas}

Desentrañar los retos que los comunicadores enfrentan a diario, así como los posibles escenarios en el mediano y largo plazo, es una responsabilidad de las carreras de comunicación cuando diseñan los perfiles de egreso y los ofertan, pues la formación debe satisfacer las demandas de la sociedad del conocimiento y del mercado sin que ello excluya el desarrollo de destrezas y habilidades de las competencias mediáticas en entornos de alta competitividad. 
Este artículo se inscribe en una perspectiva exploratoria y sus resultados se basan en un proyecto más amplio que se denomina "Tecnología, jóvenes y educación", que se llevó a cabo en la carrera de Comunicación Corporativa de la UDLA y que tiene como objetivo conocer y explicar los usos y hábitos de consumo de internet y de las TIC en los jóvenes universitarios que estudian comunicación en la ciudad de Quito en la UCE, la UDLA y la UTE. El periodo de investigación de la primera etapa de este proyecto se realizó entre los años 2015 y 2017 y se extendió el debate en la comunidad universitaria hasta $2019 .{ }^{3}$

La selección de estas universidades y sus carreras para la investigación respondió a los siguientes criterios: a) aglutinan el mayor número de estudiantes de comunicación en la capital a nivel de pregrado; ${ }^{4} b$ ) tienen una oferta académica con sus propias particularidades, lo cual enriquece la comparación; c) la UCE es pública, la UDLA es privada y la UTE es cofinanciada, por lo tanto, son tres universidades distintas; y d) en las tres se incluye la enseñanza de las TIC en correspondencia con su oferta académica. A modo informativo, cabe señalar que el Consejo de Educación Superior (CES) del Ecuador exigió nuevos enfoques en los planes de estudio de las carreras en el primer semestre de 2018 y que comenzaron a implementarse desde 2019, por lo cual, la investigación pone énfasis en la malla curricular anterior, pero que sigue todavía vigente en los semestres que concluyen las promociones en las que no se aplicaron los nuevos planes.

\footnotetext{
Entre los objetivos de este proyecto, se mencionan los siguientes: a) determinar los niveles de frecuencia y acceso a internet de los estudiantes de la UDLA, UCE y UTE en relación con los contenidos abordados en su carrera y su nivel de formación; y b) identificar los hábitos de consumo de internet en estudiantes universitarios en la producción reproducción y circulación de contenidos relacionados con su carrera.

4 A la fecha de corte de esta investigación, la Facultad de Comunicación Social de la UCE tenía 1.123 estudiantes; la carrera de Comunicación Corporativa de la UDLA, 393, y la carrera de Relaciones Públicas y Comunicación, 450.
} 
Austral Comunicación

Volumen 9, número 1 (Junio de 2020): 121-144. ISSN 2313-9129

Tabla 1. Breve descripción de las carreras de comunicación

\begin{tabular}{|c|c|c|c|}
\hline Características & UDLA & UTE & UCE \\
\hline Creación de las carreras & 2002 & 1986 & 1985 \\
\hline Tipo de universidad & Privada & Cofinanciada & Pública \\
\hline Denominación de las carreras & $\begin{array}{l}\text { Facultad de Comunicación } \\
\text { y Artes Audiovisuales }\end{array}$ & $\begin{array}{l}\text { Facultad de Comunicación, } \\
\text { Artes y Humanidades }\end{array}$ & Facultad de Comunicación Social \\
\hline Denominación de las carreras & $\begin{array}{l}\text { Facultad de Comunicación } \\
\text { y Artes Audiovisuales }\end{array}$ & $\begin{array}{l}\text { Facultad de Comunicación, } \\
\text { Artes y Humanidades }\end{array}$ & Facultad de Comunicación Social \\
\hline Perfiles de egreso & $\begin{array}{l}\text { "Administra con sentido } \\
\text { crítico las tecnologías de la } \\
\text { comunicación e información } \\
\text { en función de los objetivos } \\
\text { estratégicos de las empresas." }\end{array}$ & $\begin{array}{l}\text { "'Desarrollar una comunicación utilizando } \\
\text { medios modernos y TIC que revelan el uso } \\
\text { aceptable de las ayudas visuales (por ejemplo, } \\
\text { PowerPoint). } \\
\text { Elaborar procesos de comunicación } \\
\text { interpersonales, intergrupales y masivos } \\
\text { utilizando tecnologías informáticas } \\
\text { con iniciativa acordes a los intereses de } \\
\text { una organización y sus colectivos."'" }\end{array}$ & $\begin{array}{l}\text { "Diseña productos de } \\
\text { comunicación social para los } \\
\text { diferentes medios, con propuestas } \\
\text { coherentes y motivadoras, que } \\
\text { representan la realidad objetiva } \\
\text { sobre bases epistemológicas } \\
\text { de las ciencias sociales y de la } \\
\text { comunicación, con una amplia } \\
\text { formación cultural-humanística, } \\
\text { demostrando respeto a los } \\
\text { diversos actores sociales." }\end{array}$ \\
\hline $\begin{array}{l}\text { Materias que contemplan la } \\
\text { malla de estudios en TIC }\end{array}$ & $\begin{array}{l}31 \text { Productos Comunicacionales } \\
\text { Taller Multimedia I } \\
\text { Taller Multimedia II } \\
\text { Comunicación Multimedia }\end{array}$ & $\begin{array}{l}\text { Diseño Digital I } \\
\text { Diseño Digital II } \\
\text { Presentación Multimedia } \\
\text { Taller Profesional } \\
\text { Taller Profesional II } \\
\text { Taller Profesional III } \\
\text { Taller Profesional IV }\end{array}$ & Comunicación Multimedia \\
\hline Competencias mediáticas & No se trabajan & No se trabajan & No se trabajan \\
\hline Recursos tecnológicos & $\begin{array}{l}12 \text { laboratorios } \\
330 \text { computadoras }\end{array}$ & $\begin{array}{l}4 \text { laboratorios } \\
100 \text { computadoras }\end{array}$ & $\begin{array}{l}2 \text { laboratorios } \\
72 \text { computadoras }\end{array}$ \\
\hline
\end{tabular}

Fuentes: Facultad de Comunicación Social de la Universidad Central del Ecuador [FACSO-UCE] (2016);

Universidad de Las Américas [UDLA] (2017); Universidad Tecnológica Equinoccial [UTE] (2016); Ulloa (2018a).

Elaboración propia.

La estrategia metodológica que se propone es mixta (cualitativa y cuantitativa) (Kathryn, 2009; Tonon de Toscano, 2013). Combina la revisión de la literatura acerca del tema, analiza de manera comparada las mallas curriculares de las tres universidades -específicamente en lo que respecta a las TIC e internet- y combina los resultados de esta primera etapa con entrevistas en profundidad a las autoridades de los tres centros de estudio. Además, incluye los resultados de una encuesta realizada a los estudiantes de las tres carreras de las tres universidades de todos los semestres. ${ }^{5}$ En el diseño de

\footnotetext{
5 En correspondencia con el número de alumnos de cada carrera, se aplicó el método probabilístico, es decir, el cálculo de una muestra aleatoria y representativa, bajo la aplicación de la siguiente fórmula: $n=N /\left[e^{2}(N-1)+1\right]$ con un margen de error del 5\%. Se aplicaron 295 encuestas en la UCE, 198 en la UDLA y 212 en la UTE. El número de encuestas fue proporcional al de alumnos en cada semestre en las tres universidades.
} 
los instrumentos para las entrevistas, así como para las encuestas, se incluyeron como dimensiones de análisis lo siguiente. Para el primer caso, qué piensan las autoridades acerca del papel de las TIC en el desarrollo profesional de los estudiantes, el grado de importancia que tiene la enseñanza de las TIC en la malla curricular de su carrera y la ventaja competitiva de un comunicador frente a otros profesionales en el uso de las TIC; mientras que en las encuestas se incluyeron preguntas a los estudiantes acerca de qué recursos de internet utilizan para buscar información especializada de su carrera, las herramientas que sus universidades usan para el desarrollo del aprendizaje, si el perfil de egreso de sus carreras contempla los retos y exigencias de la comunicación digital y si hay un uso de las redes sociales para actividades académicas. En este artículo, solo se incluyen las preguntas relacionadas con el tema que se aborda y asume las omisiones que pudiera haber.

Con estos insumos se configura una primera radiografía que permite triangular la información del análisis de las mallas curriculares con los criterios de las autoridades académicas y los estudiantes. En ningún momento se trata de generalizar este primer acercamiento como si fuera la realidad universitaria del Ecuador, sino más bien abrir el debate acerca de cómo se trabaja en la academia para comprender y aprender a vivir en la sociedad de la información, la comunicación y el conocimiento.

\section{Apropiación de las TIC}

En este apartado, se exponen los resultados del trabajo empírico respecto a la comparación de los perfiles de egreso en las tres carreras de las tres universidades. Además, se introducen varios fragmentos de las entrevistas realizadas en profundidad a actores clave en el tema y los datos de la encuesta aplicada a los estudiantes para conocer hasta dónde los conceptos expuestos acerca de la sociedad del conocimiento, el mundo digital y la generación de los nativos digitales encajan en los planteamientos de las carreras de comunicación que fueron seleccionadas.

Un gran porcentaje de docentes de la Facultad de Comunicación Social de la UCE, así como de las autoridades, reconoce que la malla curricular de la carrera otorga mayor importancia a la parte teórica desde una perspectiva crítica. En este sentido, analiza cualitativamente internet y las TIC antes que privilegiar su uso instrumental y todo el potencial que ello conlleva desde la creación de páginas web, blogs o aplicaciones para dispositivos móviles.

En la Facultad de Comunicación Social de la UCE hay un porcentaje significativo de docentes de la "vieja guardia" (los de mayor antigüedad) frente a un porcentaje limitado de profesores cuasi nativos digitales, por lo cual, hay distintas maneras de usar las tecnologías en las aulas. Sin embargo, esto no altera el hecho de que en los alumnos haya una actitud instrumental de la tecnología "sin pensar que el producto [mensaje] a 
Austral Gmunicación

Volumen 9, número 1 (Junio de 2020): 121-144. ISSN 2313-9129

través de las plataformas es la consecuencia de un proceso de reflexión previo" (Natalia Angulo, comunicación personal, 2 de diciembre de 2016)”.

Arriba se evidencia que un gran porcentaje de los docentes están en un nivel básico de apropiación de las TIC, utilizando las categorías de Adell (2008), es decir que las conocen, pero no las aplican ni las inscriben en el escenario de enseñanza- aprendizaje. Esto tampoco quiere decir que los profesores más jóvenes hayan dado el salto a niveles de adaptación o de una apropiación mayor en la que se produzcan contenidos y formatos desde las TIC. Para los dos segmentos de docentes, si bien puede haber un análisis de la sociedad del conocimiento, no se hace uso de las herramientas que podrían sintonizar con aquello que se reflexiona. Por lo tanto, está la iniciativa de insertar una pedagogía crítica de las TIC, pero no se llega a desarrollar una estrategia sostenida en lo conceptual, metodológico e instrumental de las competencias mediáticas.

La carrera de Comunicación Corporativa de la UDLA "establece que su ventaja competitiva se encuentra en la utilización de herramientas digitales dentro del proceso de formación de los estudiantes. Es decir que se vayan capacitando y aprendiendo dentro de entornos virtuales (Fernando Zúñiga, comunicación personal, 4 de enero de 2017)" (Ulloa, 2018b, p. 60). Esto se hace posible porque esta universidad tiene en su modelo pedagógico un objetivo profesionalizante, es decir que los estudiantes hacen en el aula las posibles tareas que demanda el mercado laboral y esto significa que sus docentes tienen un nivel de apropiación de las TIC intermedio y avanzado, pues convierten el aula en un escenario lo más parecido posible a las demandas del trabajo. Esto no quiere decir que el uso de las tecnologías vaya acompañado de un análisis crítico, pues el uso de las herramientas informáticas puede entramparse en un escenario funcional a los intereses del mercado.

En el caso de la carrera de Comunicación de la UTE, la apropiación de las TIC por parte de los docentes pasó por un proceso de transición, en el cual hubo total discrecionalidad por parte de los profesores en la incorporación de los recursos y herramientas digitales en sus clases y en la planificación curricular a otra etapa en la que la Universidad obligó a los profesores a usar herramientas -como las aulas virtualespara el registro de la asistencia, la subida de tareas y el acceso a materiales de las distintas materias por parte de los alumnos. Esta transición, si no tiene claridad hacia dónde van los docentes con la incorporación de las TIC en cuanto a su formación profesional así como al proceso de enseñanza de los estudiantes, corre el riesgo de automatizar procesos administrativos que exigen las universidades antes que potenciar los recursos informáticos para una inserción en la sociedad del conocimiento, como el acceso y uso a las bases de datos, tutoriales en formatos digitales audiovisuales y redes colaborativas con pares de otros países en distintas plataformas, entre otros. 
Una realidad que se observa en las tres universidades es que los profesores no utilizan ni potencian los espacios que ofrece internet, así como las aplicaciones de las TIC, en la creación de blogs, páginas web u otras aplicaciones. Hay una tradición de consumo más que de producción de información. Esto no significa que los docentes atraviesen una situación de tecnofobia ni de analfabetismo digital, sino más bien que en el caso de los docentes inmigrantes digitales entraron en la zona de mayor comodidad o de navegación en la red como una rutina antes que de exploración de los recursos que mejorarían su acceso al conocimiento.

En las tres carreras se reitera el mismo fenómeno en la relación docente-estudiante: dicta clase un mayor porcentaje de profesores considerados inmigrantes digitales a estudiantes nativos digitales. Bajo esta realidad, se produce el encuentro de dos generaciones condicionadas de distinta manera por la tecnología, sin embargo, esto no configura un escenario de superioridad/inferioridad, ya que el acceso, el uso y los hábitos de consumo de internet y de las TIC por parte de los estudiantes no van de la mano de criterios de selección adecuada de información o de altos niveles de calidad, tampoco con un previo pensamiento crítico.

En este sentido, la presencia del docente sigue siendo clave en la construcción de sentido de la tecnología y sus usos.

Yo no me dejaría impresionar [de los nativos digitales]. Los chicos siguen haciendo un uso súper básico de la plataforma [internet], yo no sé cómo es la realidad en las otras universidades. Mi preocupación es cómo conceptualizas el trabajo en el entorno digital, es decir, cuál es el proceso previo (Natalia Angulo, comunicación personal, 2 de diciembre de 2016).

No obstante, también hay otra perspectiva y es la de dejar que los estudiantes, desde una lógica instrumental, sobrepasen al profesor bajo una falsa experticia en el uso de las TIC.

\begin{abstract}
Para mejorar el proceso de enseñanza-aprendizaje, hay que primero educar a los profesores [...] de mi experiencia que he tenido la oportunidad de compartir con profesores de comunicación de prácticamente toda América Latina y también en países de habla inglesa, el gran problema es que son los profesores el primer obstáculo a la enseñanza de materias digitales en las carreras y básicamente es un tema de ignorancia y de mantenerse en su zona de confort (Albertina Navas, comunicación personal, 16 de diciembre de 2016).
\end{abstract}

Este escenario complejiza aún más la realidad que se analiza, porque introduce elementos de análisis que deben ser profundizados en mayor medida. Tampoco hay respuestas determinísticas, pues cada docente es un mundo, pero sí hay realidades comunes en el sentido de que la apropiación de las TIC cobraría mejores resultados cuando esté acompañada del desarrollo de competencias mediáticas. Es decir que no 
se trata solo de formar en el uso de la tecnología, sino también en la construcción de sentidos y conceptos que se procesan y circulan a través de ella.

Tabla 2. Apropiación de las TIC

\begin{tabular}{llll}
\hline & UDLA & UTE & UCE \\
\hline $\begin{array}{l}\text { Nivel de apropiación de las TIC } \\
\text { por parte de los docentes }\end{array}$ & Intermedio & Básico & Básico \\
\hline $\begin{array}{l}\text { Manejo de competencias } \\
\text { mediáticas }\end{array}$ & No hay evidencia & No hay evidencia & No hay evidencia \\
\hline Uso de las TIC & $\begin{array}{l}\text { Docencia y } \\
\text { administrativo }\end{array}$ & Administrativo & Administrativo \\
\hline
\end{tabular}

Fuentes: FACSO-UCE (2016); UDLA (2017); UTE (2016); Ulloa (2018a). Elaboración propia.

En lo que corresponde a las competencias del perfil de egreso de las tres universidades y la importancia que tienen las TIC, se pueden constatar realidades diferenciadas en las tres universidades. Para el caso de la UCE, se observan competencias generales y específicas debido a los énfasis de graduación que tienen los estudiantes. ${ }^{6}$ Para el perfil de egreso con énfasis en comunicación organizacional, la carrera describe:

El Profesional en Comunicación Social es un comunicador capaz de diseñar, realizar, utilizar y evaluar procesos, proyectos y productos comunicativos sobre las diversas esferas de la vida, con fines sociales y empresariales, sobre la base de procesos investigativos, fundamentos epistemológico-culturales, destrezas tecnológicas y diversidad mediática [...] (FACSO-UCE, 2016, p. 17).

Respecto a las competencias específicas que constan en el mismo perfil, se menciona que: "[el estudiante] Emplea los recursos técnicos para la producción de la información: audiovisuales, multimedia y el ambiente WEB” (FACSO-UCE, 2016, p. 18). También se mencionan "destrezas tecnológicas y diversidad mediática".

Para el caso de la UTE, las competencias del perfil de egreso evidencian una mayor relevancia en comparación con la UCE en cuanto al uso instrumental de las tecnologías desde una óptica de praxis profesional y de potenciar ciertas habilidades en los estudiantes. La carrera busca "desarrollar una comunicación utilizando medios modernos y TIC que revelan el uso aceptable de las ayudas visuales (por ejemplo, PowerPoint)" y "laborar procesos de comunicación interpersonales, intergrupales y masivos utilizan-

6 Los énfasis de graduación posibilitan a los estudiantes inclinarse por algunos campos profesionales de su interés y afinidad, como, por ejemplo, la comunicación organizacional, el periodismo, el desarrollo, etcétera. 
do tecnologías informáticas con iniciativa acordes a los intereses de una organización y sus colectivos" (UTE, 2016).

La UDLA, a diferencia de sus pares, utiliza resultados de aprendizaje (RDA) antes que competencias. En su perfil de egreso, se menciona que: "[el estudiante] Administra con sentido crítico las tecnologías de la comunicación e información en función de los objetivos estratégicos de las empresas" (UDLA, 2017, p. 1). Al igual que la UTE, el énfasis sintoniza con el desempeño profesional que tendrán los estudiantes en el mundo del mercado empresarial. Un dato diferenciador es el uso obligatorio de aulas virtuales.

En los tres casos, las TIC son concebidas como recursos y medios que deben utilizarse con destreza y sentido crítico, más que como objetos de estudio que configuran y remodelan las mediaciones entre los sujetos desde entornos online, donde no se requiere interacción personal y movilidad desde un lugar hacia otro. Incluso, los flujos de información pueden viajar y "viralizarse" a la distancia de un clic desde un dispositivo móvil. Como mencionan Salinas (2001) y Selwyn (2013), estos dispositivos han creado y evidenciado un mundo más conectado, pero, además, esta conexión no es aburrida porque debe entretener al sujeto en todo momento, no solo en los de ocio.

En las tres carreras, el perfil de egreso es profesional porque busca fortalecer el desempeño de los estudiantes en el mercado laboral mediante competencias y destrezas que pasan por un alto componente de análisis crítico, como es el caso de la UCE, eficaz manejo instrumental de las TIC y el diseño de estrategias de comunicación, como propone la UDLA, y el uso de las relaciones públicas y la publicidad, como ofrece la UTE. Por ello, la oferta académica de las tres no configura un escenario de competencia entre ellas, ya que persiguen objetivos diferentes. Cabe decir que la formación es más humanística en la UCE, pues tiene un 14\% más de materias de este tipo en comparación con la UDLA y el 5\% más que la UTE.

La enseñanza y el aprendizaje en las universidades también se explican, aunque no sea un factor concluyente, en aspectos como la oportunidad, el acceso y la conectividad que tienen los estudiantes de las TIC, entre los más importantes. Por ejemplo, en lo que se refiere al acceso, en la UCE hay dos laboratorios y setenta y dos computadoras (entre PC y MAC) para un número mayor a los mil estudiantes, en la UTE hay cuatro laboratorios y cien computadoras (entre $\mathrm{PC}$ y $\mathrm{MAC}$ ) para un aproximado de cuatrocientos estudiantes y en la UDLA hay doce laboratorios y trescientas treinta computadoras (entre PC y MAC) para un número similar a la anterior universidad. En la UDLA, los equipos no son de uso exclusivo de la carrera de Comunicación Corporativa, pues son compartidos con estudiantes de otras facultades. En los tres casos, hay un acceso diferenciado, ya que en la UCE trece alumnos ocupan una computadora; en la UTE, cuatro; y en la UDLA, casi uno por cada equipo. 
Por lo tanto, entre las carreras de comunicación de las tres universidades hay diferencias, no solo en la malla curricular y en la apropiación de las TIC por parte de los docentes, sino también en el acceso que tienen los estudiantes a las computadoras. A ello habría que añadir que no hay claridad en ninguna de las tres acerca del papel que juegan las herramientas digitales, pues si bien las autoridades académicas coincidieron en que no puede soslayarse la manera en cómo estas han reconfigurado el mundo en diversos ámbitos, no basta solo con incluir en los perfiles de egreso y programas de estudio un conjunto de materias que fortalecen el manejo instrumental de las tecnologías (hardware y software) para producir piezas comunicacionales, sino que hay un vacío en cuanto al debate de lo que significa la sociedad de la comunicación, el conocimiento y la información.

Por otra parte, los perfiles de egreso de las carreras de comunicación analizadas, la formación de los docentes, el modelo de enseñanza, la metodología de apropiación de las TIC y los recursos que se utilizan como medios didácticos se inscriben en una dinámica cambiante en diversas intensidades y formas como característica del mundo globalizado. En ese sentido, las ventajas competitivas que pudiera tener un estudiante de comunicación al culminar sus carreras van de la mano de varios factores que se articulan entre sí, como la comprensión de la sociedad del conocimiento, el uso de las TIC desde una perspectiva que escape a la mera instrumentalidad y la incorporación de las competencias mediáticas para que el proceso de producción, circulación y distribución de contenidos sea pedagógico y también ético.

\section{Desde la orilla de los estudiantes de comunicación}

El proceso de enseñanza y aprendizaje no inicia ni termina solo en el papel que desempeñan los docentes, las autoridades de las universidades y las exigencias del mercado laboral, ya que pasa también por el papel que cumplen los estudiantes. En ese sentido, hay varias entradas desde las cuales se podrían obtener respuestas de los jóvenes acerca de sus experiencias y percepciones sobre cómo los centros de formación universitaria conciben los procesos educativos de las TIC en la sociedad del conocimiento.

Una primera entrada es conocer cómo es el acceso a internet y aquí se presenta un panorama descriptivo. En las tres universidades, los estudiantes usan la red los siete días de la semana, pero en lo que se refiere al uso en temas educativos, los resultados difieren. El 56\% de los estudiantes de la UCE lo hace entre cuatro y ocho horas; el $61,7 \%$ de la UTE lo hace entre una y tres horas; y el 52,5\% de la UDLA, entre una y tres horas. La primera actividad en el acceso a internet son las redes sociales, donde encabeza la UTE; y en segundo lugar, la UDLA y la UCE. Esto significa que la relación social de los estudiantes con el mundo externo es digital y desde entornos informales como las redes, en las cuales circula información sin ningún filtro y que, en la mayoría 
de los casos, se llega a compartir sin verificación ni contrastación informativa. Para los estudiantes de comunicación de las tres universidades, la actividad de consultar información ocupa el primer lugar, el 64\% en la UCE, el 69\% en la UDLA y el 73\% en la UTE, es decir, los jóvenes no entran en la categoría de prosumidores (o quienes consumen y producen información).

Aunque no haya un criterio definitivo acerca de cuáles son las influencias que tienen los estudiantes de las tres carreras de comunicación para consultar la información en internet y las redes, en la encuesta que se realizó en las tres universidades se encontraron varias pistas acerca de si los docentes tienen alguna incidencia en los hábitos de consulta de información de sus alumnos. En la encuesta, el $48 \%$ de los estudiantes de la UCE dijo que "de vez en cuando" tienen guía del docente en la utilización de internet; el $80 \%$ de la UDLA y el 36\% de la UTE respondieron lo mismo. Apenas el 19\% de la UCE dijo "casi siempre", el $41 \%$ de la UDLA y el 36\% de la UTE dijeron lo mismo. Le sigue como respuesta el $31 \%$ de la UCE que dijo "casi nunca" y lo mismo respondieron el $34 \%$ de la UDLA y el 36\% de la UTE (Ulloa, 2018a, p. 59).

Como se puede observar, el rol del docente no es determinante cuando los estudiantes consultan, investigan, procesan y circulan información, lo cual estaría relacionado con el hecho de que tampoco hay en el aula una incorporación sostenida de las TIC e internet, pero también eso implicaría que está en ciernes la incorporación de las competencias mediáticas en el perfil de egreso para una formación más amplia de los alumnos. Al respecto, habría que seguir explorando a futuro a qué planes, programas y proyectos de formación tienen acceso los docentes y en qué nivel de apropiación de las tecnologías están.

Entre los resultados de la encuesta mencionada, la información sobre las actividades que realizan los estudiantes de las carreras de comunicación en internet acerca de tres acciones concretas para su formación académica -como compartir información, consultar información y producir y compartir información (prosumidores)-, los hallazgos revelan una actitud pasiva antes que proactiva por parte de los tres grupos. Apenas el 26\% de los estudiantes de la UCE produce y comparte información, lo propio hace el 19\% de la UDLA y, en el mismo porcentaje, la UTE. Esto significa que el acceso a las TIC no garantiza producción de conocimientos, sino que esta generación responde a otras variables, ya que los estudiantes de la UCE son quienes tienen menos acceso a recursos tecnológicos, pero producen más y usan de manera más eficiente el tiempo en internet en lo que se refiere a su formación profesional.

Los estudiantes creen que el perfil de egreso de las tres carreras sí cumple con las exigencias de la comunicación digital. Esto respondió el 76\% de los estudiantes de la UDLA, el 68\% de la UTE y el 50\% de la UCE. Estos porcentajes se explicarían por la orientación que tienen las universidades en fomentar el uso de las tecnologías desde 
sus espacios de laboratorios de computación, el uso de las aulas virtuales por parte de los docentes y la impartición de algunas asignaturas en modalidad online, sin perder de vista que hay programas de estudio con modalidad a distancia en diversas facultades y carreras. En este mismo contexto, en las tres carreras se imparten materias con modalidad en línea. " Es prácticamente previsible para el futuro a corto plazo, la oferta por parte de las universidades de programas completamente acreditados en modalidades de aprendizaje con el uso de componentes digitales" (Sandia, Luzardo y AguilarJiménez, 2019, p. 269).

La percepción que tienen los docentes y los estudiantes de las tres universidades acerca de los temas tratados como dimensiones de la investigación no siempre coinciden, ya que se confrontan el deber ser del primero y las aspiraciones del segundo. Hay una tensión que no entra en conflicto, porque el rol que cada uno cumple en la coyuntura rebasa la resolución de lo estructural, es decir, de qué manera las carreras de comunicación de las universidades conciben la enseñanza de las TIC en la sociedad de la información, la comunicación y el conocimiento.

\section{Conclusiones}

La formación de los comunicadores en las tres universidades sobre la concepción y el uso de internet, así como de las TIC, es diferenciado, ya que si bien en ningún plan curricular hay un posicionamiento teórico respecto a la sociedad del conocimiento, del mundo digital y de las tecnologías, en las mallas curriculares se trata de potenciar las destrezas y habilidades técnicas de los estudiantes en el uso de los dispositivos de manera instrumental. Esta situación puede configurar un escenario pragmático más que crítico, no obstante, se observa la necesidad de replantear a futuro los perfiles de egreso, según los mandatos de la Ley Orgánica de Educación Superior (LOES).

La enseñanza de las TIC se circunscribe a un escenario multifactorial, en el cual inciden la visión de las universidades respecto al mundo contemporáneo, el perfil de egreso, la formación de los docentes, los conocimientos de los estudiantes, los recursos materiales y tecnológicos y las leyes de educación superior, pero, además, la flexibilidad de quienes lideran los procesos educativos para sintonizar las demandas y exigencias del contexto. En las tres universidades, el nivel de apropiación de las TIC evidencia un nivel básico de los docentes, ya que no incorporan en las aulas ni en su programación conceptual y metodológica las tecnologías de la información, no para sustituir la pizarra tradicional con una presentación de Power Point, sino más bien para proponer nuevas formas de consultar información, compartir desde dinámicas de inteligencia

En Ecuador, la Ley Orgánica de Educación Superior (2010) exige que las universidades promuevan "la creación desarrollo, transmisión y difusión de la ciencia, la técnica, la tecnología”, como menciona el artículo 13. 
colectiva-colaborativa y producir conocimientos en sintonía con los temas que se debaten en el mundo sin perder de vista las exigencias locales.

En las carreras de comunicación hay un porcentaje importante de profesores inmigrantes digitales y otros que están más cercanos a los cambios tecnológicos. Esto produce un escenario de doble vía. Uno, en el que los estudiantes cuentan con métodos, recursos y dinámicas tradicionales, y otro, un espacio en el que los alumnos reciben clases de profesores que innovan las dinámicas de aprendizaje. Esto tampoco quiere decir que la totalidad del alumnado cuente con las mismas condiciones en cuanto al acceso, uso e incorporación de las TIC en su formación profesional. No se puede generalizar que los millennials son un todo, pues sus condiciones socioeconómicas determinan también su desempeño académico. Pese a que las universidades abren espacios de conectividad y de acceso a recursos digitales, el número de equipos por estudiante en cada carrera de comunicación es limitado.

En las tres universidades no se han incorporado asignaturas que desarrollen las competencias mediáticas, de ahí que haya un vacío pedagógico en el proceso de producción de contenidos en lo que se refiere a fortalecer, primero, el análisis de contenido, y después potenciar los recursos de las TIC, además de analizar la proliferación de las fake news y el mundo del big data para la toma de decisiones. Esta situación pone en desventaja a los comunicadores, porque no podría reducirse el proceso de enseñanza y aprendizaje en las carreras de comunicación al uso de software y hardware sin sostener las piezas comunicacionales con contenido de calidad.

La evidencia determina que docentes y estudiantes consumen información y la comparten, pero muy pocos producen y entablan redes colaborativas más sostenidas. No hay un escenario propicio para el prosumidor. Hay un papel más pasivo. Esta realidad que puede ser similar a otros entornos abre la discusión acerca de la escala de matices que podría haber en el abordaje y la profundización de los usos y hábitos de consumo de internet en el ámbito educativo; en este caso, el universitario a nivel de pregrado.

Si el acceso a las redes sociales está entre los dos primeros lugares en cuanto al uso que los estudiantes hacen de internet todos los días, es un reto en la academia experimentar si a través de este tipo de espacios, que fueron concebidos para el entretenimiento, puede lograrse una pedagogía creativa y de inteligencia colectiva. Es decir, una propuesta que pueda resignificar el uso de las redes en una iniciativa colaborativa, colectiva, crítica y de libre circulación de pensamiento.

Las condiciones en las que se produce una inmersión en la sociedad del conocimiento por parte de las universidades va de la mano de aspectos medibles, como la oportunidad de acceso a la tecnología, la conectividad en términos de velocidad y la capacidad de almacenamiento, el acceso a fuentes de calidad -como son las bases de 
Austral Comunicación

Volumen 9, número 1 (Junio de 2020): 121-144. ISSN 2313-9129

datos y repositorios digitales-, el tipo de acceso a internet (gratuito, condicionado a pago o condicionado al acceso de ciertos sitios web); sin embargo, uno de los mayores retos es la formación de los docentes. La idea es observar qué y cómo producen contenidos para sus clases y en qué formatos, cómo gestionan sus investigaciones con otros pares desde lo online y la manera en cómo mejoran el uso de fuentes de calidad.

\section{Referencias}

Acedo, S., Lazo, C. y Aparici, R. (2013). Valores de la formación universitaria de los comunicadores en la sociedad digital: más allá del aprendizaje tecnológico, hacia un modelo educomunicativo. Razón y Palabra, (81), 1-33. En http://www. razonypalabra.org.mx/N/N81/V81/13_OsunaLazoAparici_V81.pdf.

Adell, J. (2008). Competencia digital de los profesores. Obtenido el 30 de septiembre de 2018 de www.youtube.com/watch?feature=player_embedded\&v=sLLlwJcQ-

Apolo, D., Bayés, M. y Hermann, A. (2016). Cambios educativos en los procesos de lectura digital: la pedagogía del ciberespacio como estrategia de procesamiento de contenidos en la era de internet. Redes.com, 12, 223-239.

Asamblea Nacional de Ecuador (2010). Ley Orgánica de Educación Superior (LOES). Registro Oficial Suplemento 298. Quito. Corte Constitucional.

Bauman, Z. (2006). Vida líquida. Barcelona: Paidós.

Berners-Lee, T. y Fischetti, M. (2000). Tejiendo la red: el inventor del world wide web nos descubre su origen. España: Siglo XXI.

Bianchi, M. (Comp.) (2017). Recuperar la política. Agendas de innovación política en América Latina. Buenos Aires: Asuntos del Sur - Democracia en Red.

Bisbal, M. (2001). La nueva escena y el comunicador social: el resentimiento entre la "razón edificante" y la "razón instrumental" ¿Desde dónde pensamos el problema? Diálogos de la Comunicación, 62, 8-25.

Buckingham, D. (2007). Digital Media Literacies: rethinking media education in the age of the Internet. Research in Comparative and International Education, 2(1), 43-55.

Cassany, D. y Ayala, G. (2008). Nativos e inmigrantes digitales en la escuela. CEE Participación educativa, 9(4), 53-71.

Castells, M. (1997). La era de la información: economía, sociedad y cultura. (Vol. 1). Madrid: Alianza Editorial.

Castells, M. (2009). Comunicación y poder. Madrid: Alianza Editorial.

Durán Barba, J.y Nieto, S. (2017). La política en el siglo XXI. Arte, mito o ciencia. Buenos Aires: Debate.

Facultad de Comunicación Social de la Universidad Central del Ecuador. (2016). Malla curricular. Obtenido el 20 de octubre de 2019 de http://www.uce.edu.ec/web/facso. 
Ferrés, J. y Piscitelli, A. (2012). La competencia mediática: propuesta articulada de dimensiones e indicadores. Comunicar, (38), 75-82. doi: 10.3916/C38-2012-02-08.

Ficarra, F. (2001). ¿Qué deben hacer las Escuelas de Comunicación? Chasqui, 74, 14-19.

García, M., González, V. y Ramos, C. (2010). Modelos de interacción en entornos virtuales de aprendizaje. Tonos digital, 19, 1-27.

González, A. y De Pablos Pons, J. (2015). Factores que dificultan la integración de las TIC en las aulas. Revista de Investigación Educativa, 33(2), 401-417. doi: 10.6018/ rie.33.2.198161.

Gutiérrez-Rubí, A. (2016). Millennials en Latinoamérica Una perspectiva desde Ecuador. Barcelona: Ariel y Fundación Telefónica.

Hooper, S. y Rieber, L. P. (1995). Teaching with technology. En Ornstein, A. C. (Ed.), Teaching: Theory into practice (pp. 154-170). Needham Heights, MA: Allyn and Bacon.

Instituto Nacional de Estadística y Censos (INEC) (2017). Hablando de millennials. Obtenido el 20 de julio de 2019 de https://www.ecuadorencifras.gob.ec/ documentos/web-inec/Inforgrafias-INEC/2017/millenials.pdf.

Kathryn, P. (2009). Diseño de metodologías mixtas. Una revisión de las estrategias para combinar metodologías cuantitativas y cualitativas. Revista Renglones, 60, 37-42.

Linne, J. (2014). Now, I do Academic Fast Food. College Students in the Internet. Palabra Clave, 17(3), 695-716. doi: 10.5294/pacla.2014.17.3.6.

López, L. y Aguaded, M. (2015). La docencia sobre alfabetización mediática en las facultades de Educación y Comunicación. Comunicar, XXII(44), 187-195.

Marín, I., Rivera, D. y Celly, S. (2014). Estudio sobre formación en competencia audiovisual de profesores y estudiantes en el sur de Ecuador. Cuadernos.Info, (35), 119-131. doi: 10.7764/cdi.35.628.

Naím, M. (2013). El fin del poder. Barcelona: Debate.

Negroponte, N. (1995). El mundo digital. Buenos Aires: Editorial Atlántida.

Piscitelli, A. (2009). Nativos digitales. Buenos Aires: Santillana.

Prensky, M. (2001). Nativos digitales, inmigrantes digitales. Onthehorizon, 6(9), 1-7.

Punín, M. (2012). Los estudios de comunicación social/periodismo en el Ecuador. Una visión crítica al rol de la universidad y la academia. Razón y Palabra, (79), 1-22. Obtenido de http://www.razonypalabra.org.mx/N/N79/V79/66_Punin_V79.pdf Acceso: 24 de noviembre de 2019.

Punín, M., Rivera, D., y Marín, I. (2014). El periodista digital en Ecuador. Reflexiones desde la academia. Revista Dilemas Contemporáneos: Educación, Política y Valores, (1), 1-20.

Rheingold, H. (2004). Multitudes inteligentes: la próxima revolución social. Barcelona: Gedisa. 
Austral Comunicación

Volumen 9, número 1 (Junio de 2020): 121-144. ISSN 2313-9129

Rodríguez de las Heras, A. (2008). Las TIC en la educación: un proceso complejo. Ábaco, 2, 97-103.

Salinas, J. (2004). Innovación docente y uso de las TIC en la enseñanza universitaria. Revista Universidad y Sociedad del Conocimiento, 1(1), 1-16.

Salinas, J. (2011). Modelos emergentes en entornos virtuales de aprendizaje. Obtenido de http://gte.uib.es/pape/gte/sites/gte.uib.es.pape.gte/files/Modelos-emergentesen-entornos-virtuales-de-aprendizaje.Pdf Acceso: 28 de septiembre de 2019.

Sandia, B., Luzardo, M. y Aguilar-Jiménez, A. (2019). Apropiación de las tecnologías de información y comunicación como generadoras de innovaciones educativas. Ciencia, Docencia y Tecnología, 30(58), 267-289.

Sartori, G. (2010/1997). Homo Videns La sociedad teledirigida. México, D. F.: Santillana.

Selwyn, N. (2013). Internet y educación. En 19 ensayos fundamentales sobre cómo internet está cambiando nuestras vidas (196-215). España: BBVA.

Tofller, A. y Toffler, H. (2006). La revolución de la riqueza. México: Debate.

Tonon de Toscano, G. (2013). La integración de métodos de investigación en Ciencias Sociales: la triangulación y los métodos mixtos. México: El Colegio Mexiquense.

Trillos, J. (2010). Las TIC ¿endiosamiento o satanización? Análisis de su impacto en las competencias lecto-escritoras en estudiantes de Comunicación Social. Encuentros, (16), 75-86.

Ulloa, C. (2014). Comunicación: oferta académica y mercado laboral. Estrategas, 1, 53-62.

Ulloa, C.(Ed.) (2018a). Los millennials frente al espejo. Tecnología, jóvenes y sociedad. Quito: UDLA Ediciones.

Ulloa, C. (2018b). Millennials y política. En El Universo. Obtenido el 20 de octubre de 2019 de https://www.eluniverso.com/opinion/2018/07/10/nota/6851969/ millennials-politica.

UNESCO(2011).Unescoictcompetency frameworkforteachers.Paris-France.Obtenido el 20 de agosto de 2019 de http://www.unesco.org/new/en/communication-andinformation/ resources/publications-and-communication-materials/publications/ full-list/unesco-ict-competency-framework-for-teachers.

UniversidaddeLasAméricas(UDLA).(2017).Comunicación Corporativa.Obtenidoel 14 de noviembre de 2019 de http://www.udla.edu.ec/carreras/programas-academicos/ pregrados/facultad-de-comunicacion/comunicacion-corporativa-3/.

Universidad Tecnológica Equinoccial (UTE) (2016). Relaciones Públicas y Comunicación Organizacional. Obtenido el 14 de octubre de 2019 de https:// www.ute.edu.ec/facultad-de-comunicacion-artes-y-humanidades/carreras/ relaciones-publicas-y-comunicacion-organizacional.

Varela, H. (2012). Los "Y" o millennials: atributos generacionales versus perfil ideal del 
Uso de las TIC en tres facultades de comunicación ecuatorianas

extensionista contemporáneo. Estrategias para el trabajo en extensión rural. Entre Ríos: AADER-INTA.

Whitby, G. (2013). Educating Gen Wi-Fi: How We Can Make Schools Relevant for 21st Century Learners. Sidney: ABC Books.

Yoguel, G. (2014). Información y conocimiento: las vinculaciones entre difusión de tic y competencias tecnológicas. En Valenti, G. y Casalet, M. (Coords.), Instituciones, sociedad del conocimiento y mundo del trabajo (pp. 287-316). México: Flacso.

\section{Entrevistados}

Albertina Navas, experta en pedagogía en el uso de las TIC (16/12/2016).

Fernando Zúñiga, docente de la carrera de Comunicación Corporativa de la UDLA $(1 / 04 / 2017)$

Natalia Angulo, docente dela Facultad de Comunicación Social de la UCE.(02/12/2016). 